\title{
A NEW ROBOTIC SYSTEM FOR CT-GUIDED PERCUTANEOUS PROCEDURES WITH HAPTIC FEEDBACK
}

\author{
B. Maurin (1), O. Piccin (2), B. Bayle (1), J. Gangloff (1), \\ M. de Mathelin (1), L. Soler (3), A. Gangi (4).
}

March 2, 2004

(1) LSIIT, Université Louis Pasteur, 67400 Illkirch, FRANCE

(2) LICIA, INSA Strasbourg, 67000

Strasbourg, FRANCE

(3) IRCAD, 67000 Strasbourg, FRANCE

(4) Department of Radiology B., University Hospital of Strasbourg, 67091 Strasbourg, FRANCE

\begin{abstract}
In this paper, we present a new point of view for the conception of what a teleoperated robotic device should be for percutaneous intervention with computed tomography guidance. We focus our attention on very common and widely used medical acts : the radiofrequency ablation and the biopsy procedure. These acts are widely used in manual interventional radiology, but they come up with others problems. First, the guidance precision is limited for a human, second, the radiologist risks its health by being exposed to $\mathrm{X}$ rays during each operation. The robotic system we describe is original by its conception and its control feedback (based on force feedback and vision). A prototype is presented, together with a complete workflow of the system. Some results present the first data acquired with the force feedback sensors.
\end{abstract}

\section{Introduction}

\subsection{Motivation}

New minimally invasive techniques were recently developed thanks to the related progress of medical imaging and medical devices. Among these methods, percutaneous interventions offer new possibilities for therapy as well as for diagnosis. Percutaneous procedures consist in introducing a needle through the skin in order to perform local treatments on internal organs. Among these procedures, we are mainly interested in the radiofrequency ablations of tumors and in biopsies, that require high precision targeting. Radiofrequency consists in heating the tumor with a special radiofrequency-needle directly inserted inside. This intervention is less painful for the patient than a classical surgical act, and allows for faster recovery. The success of the procedure is highly correlated with the accuracy of the needle positioning. Biopsies also require high accuracy in targeting the living tissue that need to be analyzed given the small size of some tumors.

Currently, for these two techniques, the needle is hold by the radiologist. A visual guidance is needed since freehand guidance with direct tactile feedback is not sufficient. Ultrasound and X-rays imaging device (CT-scan, fluoroscope) are often used for this purpose. For very precise guidance (about 1 millimeter) computed tomography has proved to be an excellent imaging modality given its accuracy and a good tissue differentiation. During a CT-guided needle insertion, the interventionist can be exposed to high radiation of $\mathrm{X}$-rays which become dangerous for his health when he performs a lot of interventions. To avoid critical organ areas, like e.g. the portal vein in the liver, or the spinal chord, the precision of the visual guidance is crucial. Today, manual interventions are routinely made on tumors of 3 to $6 \mathrm{~cm}$, while recent CT-scans allow for the detection of tumors of $1 \mathrm{~cm}$ and below. The destruction of these tumors with freehand insertion is not possible due 
to accuracy problems. For all these reasons, CT-guided robotic systems are a very promising solution.

\subsection{Existing robotic systems}

As far as we know, the PAKY robotic system at John Hopkins University is the most advanced existing solution. Developed by Stoianovici [8], the PAKY is a one degree of freedom needle driver using a friction transmission applied to the the body of the needle. This is a remote handling tool that puts the radiologist away from the X-rays source. This tool can be attached to other handling robots or passive arms (for example, a Remote Center of Motion RCM robot with two degrees of freedom, or a positioning arm with multiple passive joints). Some methods have been developed for the automatic registration of the needle and for the visual servoing of the robot (see Navab [7], Masamune [5] and Susil [9] for the registration). The main limitations of the existing system are the lack of force feedback during insertion, a limited exerted force on the needle due to the friction, and the lack of real-time compensation of the breathing and the movements of the patient. That could be a critical problem for the safety of the patient.

Another image guided system for percutaneous intervention is the Ultrasound-guided Motion-adaptive Instrument UMI, described by Hong [3]. This robot has two degrees of freedom and is manually handled and positioned on the abdomen of the patient. It has a real-time visual servoing control loop that uses an on-board ultrasound probe for data acquisition (ultrasoundlike images). This is useful for the automatic guidance of the needle. However, the poor quality of the imaging device does not make accurate targeting easy to achieve. So, it is not suitable for tumors smaller than $1 \mathrm{~cm}$ or near bone. At last, this robot has no force feedback capabilities, mainly because it is already hold by the physician.

\subsection{Contributions}

This paper presents a novel robotic system which solves the problems of force feedback, needle position accuracy and CT-scan compatibility required in radiofrequency and biopsy interventions. The proposed robotic system aims to help the radiologist during percutaneous interventions made in the abdominal zone through a master-slave tele-operation workflow. The system is composed of a light robot that is attached to the patient body and an haptic interface. The robot offers a sufficient operating space for the needle actuation. It is a five degrees of freedom robot that accepts a special needle driving tool as its end-effector. A force sensor is positioned between the robot and the needle insertion tool for the force feedback needs. The two degrees of freedom insertion tool uses a very novel approach that removes the friction problem, keeping the translation and rotation movement actuated. A commercial haptic interface is used as a first master device.

\section{The medical constraints}

The robotic device aims to replace the arm and the hand of the radiologist in some critical steps of an intervention, in particular during X-rays visual guidance of the needle. This is to increase the safety of the physician and to give a better care for the patient. The goal is not necessarily to completely replace the physician (full automatic mode), but to provide an assistance for the needle insertion (teleoperation mode with automatic guidance). A brief survey of percutaneous procedures (see A. Gangi [2]) can give some precise constraints for the conception: small operating space, sterility, physical compatibility to X-rays, safety, mobility, accuracy and above all tactile feedback.

The operating space constraint is due to the imaging device gantry space. There is no major restriction when dealing with C-arm fluoroscopy but in the case of CT-scan, especially when the patient is obese, the space constraint is really strong: the handling device must to be contained inside a critical hemisphere of $20 \mathrm{~cm}$ around the entry point of the needle.

The sterility constraint implies that all parts of the system that can be potentially touched by the radiologist must be sterilized or packed in a sterile plastic bag.

We should avoid metal alloys inside the CT imaging device for imaging compatibility with the X-rays (diffusion). Synthetic materials are well suited.

For the safety of the patient, the robot must be quickly removable from the body or put in a movement-free mode to let the medical staff 
operate. The robot must not hamper with the radiologist, the patient, or the imaging device.

The mobility of the needle is highly depending on the type of percutaneous intervention, but we can roughly conclude that three degrees of freedom for the initial positioning of the needle at the entry point and two more degrees of freedom for the orientation are sufficient (the self spining of the needle is not made by the positioning tool but by the inserting tool).

The system must have a better accuracy than a human can achieve by hand. Thus, we would like to achieve an accuracy of $2 \mathrm{~mm}$ or less inside the patient, with a possible exertable force of 20 Newtons along the insertion axis. This corresponds to a measured maximum force that was obtained during in-vivo animal tests. The haptic sensation must have a small resolution so that the radiologist is able to feel thin transitions through membranes and changes in tissues resistance. This constraint is the novel contribution of the project in regard to the existing ones.

\section{The robotic positioning device}

Contrary to the PAKY which is connected to handling arms, we designed a robot that is positioned on the body of the patient. It weights about $2.5 \mathrm{~kg}$ and has five degrees of freedom. The robot is attached using straps as the Light Endoscopic Robot of Berkelman and Troccaz [1]. The robot is positioned on the patient in order to avoid some safety issues due to the motions of the patient with respect to the operating table.

Once this choice of a small robot had been done, a mechanical structure that corresponds to our needs was selected. Existing structures were studied with their advantages and drawbacks. We were looking for a structure that minimizes flexibility and vibrations in order to keep a good accuracy during the needle insertion. For all these reasons, we chose to have a parallel structure as the NeuroBot for skull neurosurgery. Parallel structures are used in many industrial and research applications that require rigidity, compactness, and for heavy loads. In addition, they offer a very good absolute (not only repetitive) positioning of their end-effector. Their essential drawback is the complexity of their modeling and control. We designed a structure that could be geometrically and mathematically modeled without too much difficulties. This structure is made of two 6-bars pantograph mechanisms, for which the mathematical study was made by Hunt [4]. A numerical simulation with MATLAB and Open Dynamic Engine has shown that the mathematical solution was correct and that the working space was acceptable. Since huge forces and torques are exerted on the end-effector and given the dimensions of the robot links, the active joints must support about $0.3 \mathrm{Nm}$ when stopped. The actuated axes include an ultrasonic USR-30 motor from Shinsei Corp. together with encoders and gears. These motors cannot be used to estimate the applied torques. The exerted force is measured by a force sensor with three degrees of freedom, positioned between the needle driving tool and the positioning robot. The two degrees of freedom needle driving tool introduce a new concept for holding the needle. This device uses a special blocking part that avoid any movement of the needle and that transmits all the exerted forces to the handling tool.

\section{Architecture and work- flow}

The overall system is based on a master-slave architecture. Currently, the master part is a six degrees of freedom commercial haptic device from Sensable Corp. and we are currently designing a novel haptic interface for percutaneous procedures. The slave part is the robotic (five+two degrees of freedom) device mentioned before. The workflow of a procedure is conceptually the same as proposed by Masamune [5]. The vision feedback is given by the CT-scan allowing the radiologist to verify each step in the procedure:

- Firstly, an image slice is acquired with the CT-scan and the robot position is reconstructed thanks to a stereotaxy algorithm together with a $3 \mathrm{D}$ fiducial attached to the robot (see the work of Susil [9] and a modified version in [6] with real-time computation of maximal error bounds).

- Secondly, the entry point is then either automatically selected by a planning algorithm or manually by the radiologist. 
- Thirdly, the robot is positioned at the entry point with the right orientation either automatically or manually with the haptic interface.

- Fourthly, another image slice is acquired to validate the needle trajectory. If a planning was made, the robot can check its own position by using the stereotactic fiducial.

- Finally, the robot is put in an "insertion" mode so that only the descent motions are possible. At the same time, the haptic interface is blocked to keep a line direction (two degrees of freedom) and a force control loop is used.

The radiologist may repeat this workflow as many time he needs. There is a big advantage in switching between the "positioning" and "insertion" modes. This gives extra safety on the overall system. The only critical part is the needle descent, which has one or two degrees of freedom (descent and rotation on itself). The control of this last motion is made using force feedback, in order to use the radiologist abilities to detect the type of tissues by tactile sensing.

\section{Conclusion}

We presented a novel concept for robotic percutaneous interventions: we use a parallel structure for the handling tool, together with a haptic feedback given by a force sensor. The system is teleoperated and its architecture is described. A prototype has been build and we showed some of its properties (operating space, safety, visual servoing). Experimental results will be detailed in the paper. We think that our robotic system, together with the haptic interface, will become a valuable tool that will increase the performance of percutaneous procedures.

\section{References}

[1] P. J. Berkelman, P. Cinquin, J. Troccaz, J.-M. Ayoubi, C. Létoublon, and F. Bouchard. A compact, compliant laparoscopic endoscope manipulator. In Proceedings of the 2002 IEEE International Conference on Robotics and Automation, pages 1870-1875, Washington DC, USA, May 2002. ICRA'02.

[2] A. Gangi and J.-L. Dietemann. Tomodensimétrie Interventionnelle. Editions Vigot, PARIS, 1994.
[3] J. Hong, T. Dohi, M. Hasizume, K. Konishi, and N. Hata. A motion adaptable needle placement instrument based on tumor specific ultrasonic image segmentation. In Proceedings of the 2002 Medical Image Computing and Computer-Assisted Intervention Conference, Tokyo, Japan, September 25-28 2002. MICCAI'02.

[4] K. Hunt. Kinematic Geometry of Mechanisms. Oxford Engineering Series, Clarendon Press, 1978.

[5] K. Masamune, G. Fichtinger, A. Patriciu, R. C. Susil, R. H. Taylor, L. R. Kavoussi, J. H. Anderson, I. Sakuma, T. Dohi, and D. Stoianovici. System for robotically assisted percutaneous procedures with computer tomography guidance. Computer Aided Surgery, 6:370 - 383, 2001.

[6] B. Maurin, C. Doignon, M. de Mathelin, and A. Gangi. Pose reconstruction from an uncalibrated computerized tomographic device. In Proceedings of the IEEE International Conference on Computer Vision and Pattern Recognition, volume 1, pages 455-460. CVPR 2003, June 2003.

[7] N. Navab, B. Bascle, M. H. Loser, B. Geiger, and R. H. Taylor. Visual servoing for automatic and uncalibrated needle placement for percutaneous procedures. In Proceedings of the IEEE International Conference on Computer Vision and Pattern Recognition, volume 2, pages 23272334. CVPR 2000, June 2000.

[8] D. Stoianovici, J. A. Cadeddu, H. A. B. R. D. Demaree, R. H. Taylor, L. L. Whitcomb, and L. R. Kavoussi. A novel mechanical transmission applied to percutaneous renal access. Proceedings of the ASME Dynamic Systems and Control Division, DSC-Vol. 61:401-406, 1997.

[9] R. C. Susil, J. H. Anderson, and R. H. Taylor. A single image registration method for ct guided interventions. In Proceedings of the International Conference on Medical Image Computing and Computer-Assisted Intervention (MICCAI 99), pages 798-808, 1999. 